\title{
A NOTE ON POWER INVARIANT RINGS
}

\author{
JOONG HO KIM \\ Department of Mathematics \\ East Carolina University \\ Greenville, N.C. 27834 \\ U.S.A.
}

(Recelved September 1, 1980)

ABSTRACT. Let $R$ be a commutative ring with identity and $R((n))=R\left[\left[X_{1}, \ldots, X_{n}\right]\right]$ the power serles ring in $n$ independent indeterminates $x_{1}, \ldots, x_{n}$ over $R . R$ is called power invariant if whenever $S$ is a ring such that $\left.R\left[x_{1}\right]\right] \cong S\left[\left[x_{1}\right]\right]$, then $R \cong S$. $R$ is said to be forever-power-invariant if $S$ is a ring and $n$ is any positive

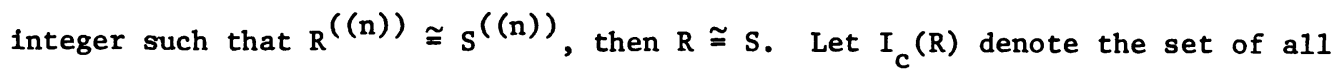
$a \in R$ such that there is $R$ - homomorphism $\sigma: R[X]] \rightarrow R$ with $\sigma(X)=a$. Then $I_{c}(R)$ is an ideal of $R$. It is shown that if $I_{c}(R)$ is $n i 1, R$ is forever-power-invariant. KEY WORDS AND PHRASES. Power series ring, Power invariant ring, Forever-powerinvariant, Ideal-adic topology.

1980 Mathematics Subject Classification Codes. $13 F 25$

1. INTRODUCTION.

In this paper all rings are assumed to be commutative and to have identity elements. Throughout this paper the symbol $\omega$ and $\omega_{0}$ are used to denote the sets, of positive and negative integers, respectively. Let $R^{((n))}=R\left[\left[x_{1}, \ldots, x_{n}\right]\right]$ be the formal power series ring in $n$ indeterminates $x_{1}, \ldots, x_{n}$ over a ring $R$ and let $\alpha_{1}, \ldots, \alpha_{n}$ be elements of $R^{((n))}$. Let $\left(R^{((n))},\left(\alpha_{1}, \ldots, \alpha_{n}\right)\right)$ denote the topological ring $R^{((n))}$ with the $\left(\alpha_{1}, \ldots, \alpha_{n}\right)$ - adic topology where $\left(\alpha_{1}, \ldots, \alpha_{n}\right)$ is the 1deal of $R^{((n))}$ generated by $\alpha_{1}, \ldots, \alpha_{n}$. It is well known that $\left(R^{((n))},\left(\alpha_{1}, \ldots, \alpha_{n}\right)\right)$ is Hausdorff if and only if $n_{j \in \omega}\left(\alpha_{1}, \ldots, \alpha_{n}\right)^{j}=(0)$. In this case, the topological ring $R^{((n))}$ is metrizable, and we say that $\left(R^{((n))},\left(\alpha_{1}, \ldots, \alpha_{n}\right)\right)$ is complete if 
each Cauchy sequence of $R^{((n))}$ converges in $R^{((n))} \cdot$ Clearly, $\left(R^{((n))},\left(X_{1}, \ldots, X_{n}\right)\right)$ is a complete Hausdorff space. If $\alpha \in R^{((n))}$, then $\alpha$ is uniquely expressible in the form $\Sigma_{j=0}^{\infty} \alpha_{j}$, where $\alpha_{j} \in R\left[X_{1}, \ldots, X_{n}\right]$ for each $j \in \omega_{0}$ such that $\alpha_{j}$ is 0 or a homogenous polynomial (that is form) of degree $j$ in $x_{1}, \ldots, x_{n}$ over $R$. We call $\Sigma_{j=0}^{\infty} \alpha_{j}$ the homogenous decomposition of $\alpha$, and for each $j \in \omega_{0}, \alpha_{j}$ is called the $j$-th homogenous component of $\alpha$.

Coleman and Enochs [3] raised the following question: Can there be non-isomorphic rings $R$ and $S$ whose polynomial rings $R[X]$ and $S[X]$ are isomorphic? Hochster [8] answered the question in the affirmative. The analogous question about formal power series rings was raised by 0 'Malley [13]: If $R[[X]] \cong S[[X]]$, must $A \cong B$ ? Hermann [7] showed that there are non-1somorphic rings $R$ and $S$ whose formal power series ring $R[X]]$ and $S[X]]$ are isomorphic. Then what is necessary and sufficient conditions on a ring $R$ in order that whenever $S$ is a ring such that $R[X]] \cong S[X]]$, then $R \cong S$ ? Several authors $[7,10,13]$ investigated sufficient conditions on $R$ so that $R$ should be power invariant, but we do not know the necessary conditions on $R$. The fact that rings with nilpotent Jacobson radical are power invariant is known in [10] and Hamann [7] proved that a ring $R$ is power invariant, if $J(R)$, the Jacobson radical of $R$, is nil. In this paper we impose more relaxed condition on $J(R)$ so that $R$ should be power invariant and forever-powerinvariant. Let $I_{c}(R)$ denote the set of all $a \in R$ such that there is an R-homomorphism $\sigma: R[X]] \rightarrow R$ with $\sigma(X)=a$. Then $I_{C}(R)$ is an ideal of $R$ contained in $J(R)$ and contains the nil-radical of $R$ (by Theorem $E,[4]$ ). Then $I_{c}(R)$ may be properly contained in $J(R)$ and it may properly contain the nil-radical of $R$. For example, if $A=Z /(4)[X]$, then $M=(2, X)$ is a maximal ideal of $A$. Let $R=A_{M}[[Y]]$, then the nil-radical of $R$ is $2 R$ and $I_{C}(R)=(2, Y)$ and $J(R)=(2, X, Y)$. Also it is easy to see that the nil-radical of $A_{M}$ is (2) and $I_{C}\left(A_{M}\right)=(2)$ and $J\left(A_{M}\right)=(2, X)$. This shows that for some ring $R, I_{c}(R)$ is nil, but $J(R)$ is not nil. It is well known that $J(R((n)))=J(R)+i_{i=1}^{n} X_{i} R^{((n))}$. Analogously, the following relation was proved in [6]: $I_{c}(R((n)))=I_{c}(R)+\sum_{i=1}^{n} x_{i} R^{((n))}$; therefore, for any ring $R$ and any positive integer $n, I_{c}(R((n))$ ) can not be nil. 


\section{SOME POWER INVARIANT RINGS.}

Let $\alpha=\sum_{i=0}^{\infty} a_{i} X_{i} \in R[[X]]$. If $n_{n=1}^{\infty}\left(a_{0}^{n}\right)=(0)$ (or $n_{n=1}^{\infty}\left(\alpha^{n}\right)=(0)$ ) and $R$ is complete with respect to the $\left(a_{0}\right)$-adic topology (or $R[[X]]$ is complete with respect to the (a)-adic topology), then there is an R-endomorphism $\phi$ of $R[X]]$ such that $\phi(X)=\alpha,([14]$ and [15]).

The following theorem from [15] will be needed for our main results.

THEOREM 1. Let $\alpha=i_{i=0}^{\infty} a_{i} X^{i} \in R[[X]]$. Then there exists an R-automorphism $\phi$ of $R[X]]$ such that $\phi(X)=\alpha$ if and only if the following conditions are satisfied:

(1) $(R[X]],(\alpha))$ is a complete Hausdorff space;

(2) $a_{1}$ is a unit of $R$.

The next theorem (Theorem 5.6, [5]) is the more generalized form of Theorem 1. THEOREM 2. Let $\alpha_{i}=\stackrel{\infty}{=}=0_{j}^{(i)} \in R^{((n))}$ for $i=1, \ldots, n$, be homogeneous decompositions of elements of $R^{((n))}$. There exists an R-automorphism $\phi$ of $R^{((n))}$ such that $\phi\left(\mathrm{X}_{1}\right)=\alpha_{i}$ for each 1 if and only if the following conditions are satisfied:

(1) $\quad\left(R^{((n))},\left(\alpha_{1}, \ldots, \alpha_{n}\right)\right)$ is a complete Hausdorff space;

(2) $\mathrm{R} \alpha_{1}{ }^{(1)}+\ldots+\mathrm{R} \alpha_{1}{ }^{(n)}=\mathrm{R} \mathrm{x}_{1}+\ldots+\mathrm{R} \mathrm{x}_{\mathrm{n}}$.

Moreover, if such an automorphism $\phi$ exists, then it is unique.

A1so, we need the following proposition:

PROPOSITION 3. Let $M$ be a unitary free R-module of finite rank $n$ and let $\mathrm{n}$ $\left\{x_{i}\right\}{ }_{i=1}^{n}$ be a free basis for $M$. Let $M_{n}(R)$ denote the ring of $n \times n$ matrices over $R$, and let $z_{1}, \ldots, z_{n}$ be elements of $M$ such that $z_{i}=\sum_{j=1}^{n} a_{i j} x_{j}$ for each $i=1, \ldots, n$ where $a_{i j} \in R$ for each $i$. and $j$. Then the following conditions are equivalent:

(1) $R z_{1}+\ldots+R z_{n}=R x_{1}+\ldots+R x_{n}$

(2) $\operatorname{det}(A)$, the determinant of $A$, is a unit of $R$ where $A=\left(a_{1 j}\right)$ is the n $x$ n matrix.

(3) $\left\{z_{i}\right\}_{i=1}^{n}$ is a free basis for $M$.

The proof of the proposition is stralghtforward so we omit its proof.

Finally, we list the theorem from [4] which plays a particularly important role in this paper. 
THEOREM 4. Let

$I_{1}=\{a \in R \mid$ there exists an R-automorphism $\left.\sigma: R[X]] \rightarrow R[X]\right]$ with $\sigma(x)=X+a$ and $I_{2}=\left\{a \in R \mid\right.$ there exists an $R$-homomorphism $\sigma: R\left[\left[\mathrm{X}_{1}, \ldots, \mathrm{X}_{\mathrm{n}}\right]\right] \rightarrow \mathrm{R}\left[\left[\mathrm{Y}_{1}, \ldots, \mathrm{Y}_{\mathrm{m}}\right]\right]$ such that $\sigma\left(X_{1}\right)=a+f$ for some $X_{i}$ and $\left.f \in \Sigma_{j=1}^{m} Y_{j} R\left[\left[Y_{1}, \ldots, Y_{m}\right]\right]\right\}$.

Then $I_{c}(R)=I_{1}=I_{2}$.

Now we are ready for our first result.

THEOREM 5. If $R$ is a ring such that $I_{c}(R)$ is $n i l$, then $R$ is power invariant.

PROOF. Suppose that $I_{c}(R)$ is nil. Let $\phi$ be an isomorphism of $\left.R[X]\right]$ onto $S[[X]]$. Then $\phi(R)[[\phi(X)]]=S[[X]]$; therefore, in order to show power invariance of $R$, it suffices to show that $R[X]]=S[Y]]$ implies $R \cong S$, where $Y$ is an indeterminate over a ring $S$. Let $W=R[X]]=S[Y Y]$ and let $Y=a_{0}+X U$ and $X=b_{0}$ $+Y V$ where $a_{0} \in R, b_{0} \in S$ and $U, V \in W$. Clearly $(W,(Y))$ is a complete Hausdorf $f$ space; therefore, there is an R-endomorphism $\sigma$ of $R[X X]$ such that $\sigma(X)=Y=a_{0}$ $+\mathrm{XU}$. Then by Theorem 4, $a_{0} \in I_{c}(R)$ and so $a_{0}$ is a nilpotent element of $R$. Let $a_{0}=\sum_{1=0}^{\infty} c_{i} Y^{1}$ where $c_{i} \in S$ for each $i \in \omega_{0}$, then $c_{i}$ is nilpotent for each $i \in \omega_{0}$ and we have

$$
Y=\stackrel{\sum}{\Sigma}=0_{1}^{\infty} c^{1}+b_{0} U+Y v U
$$

The $Y$ coefficients in both sides of (1) yields $1=c_{1}+b_{0} u_{1}+v_{0} u_{0}$ where $u_{0}$ and $v_{0}$ are constant terms of $U$ and $V$ considered as elements of $S[Y Y]$, respectively and $u_{1}$ is the $Y$ coefficient of $U$ considered as an element of $S[Y Y]$. Since $X$ is an element of $J(R[X]])=J(W), b_{0}+Y V$ is an element of $J(S[[Y]])$ and so $b_{0}$ is an element of $J(S)$. Recall that $c_{1}$ is a nilpotent element of $s$, then $c_{1}+b_{0} u_{1} \in J(S)$; therefore, $v_{0} u_{0}=1-c_{1}-b_{0} u_{1}$ is a unit of $s$. This forces $U$ and $v$ to be units of $W=S[Y Y]]$. If we consider $U$ as an element of $R[X]]$ and let $U={ }_{i=0}^{\infty} a_{i+1} X^{1}$, $a_{1+1} \in R$ for each $1 \in \omega_{0}$, then the constant term $a_{1}$ is a unit of $R$. Then $Y=\sum_{1=0}^{\infty} a_{1} X^{1}$ where $a_{1}$, the $X$ coefficient, is a unit of $R$, and $(W,(Y))$ is a complete Hausdorff Space. Then by Theorem 1 , there exists an R-automorphism $\psi$ of $R[[X]]$

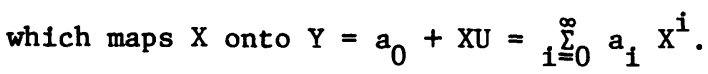

Then $R \cong R[[X]] /(X) \cong W /\left(a_{0}+X U\right)=W /(Y) \cong S$. This completes the proof.

Let $R[t]$ be the polynomial ring in an indeterminate $t$ over a ring $R$, then $J(R[t])$ coincides with the nil-radical of $R[t]$; therefore, $I_{c}(R[t])$ is a nil ideal 
of $(R[t])$ and by Theorem $5, R[t]$ is power invariant. Similarly, if $R\left[t_{1}, \ldots, t_{n}\right]$ is the polynomial ring in $n$ indeterminates $t_{1}, \ldots, t_{n}$ over $R$, then $R\left[t_{1}, \ldots, t_{n}\right]$ is power Invariant.

It is natural to ralse the following question: For what kind of $r i n g R$, is $R$ isomorphic to $S$ whenever $R\left[\left[x_{1}, \ldots, x_{n}\right]\right]$ and $s\left[\left[x_{1}, \ldots, x_{n}\right]\right]$ are isomorphic for some positive integer $n$ ? To wit, we give the following definition.

DEFINITION. A ring $R$ is said to be forever-power-invariant provided $R$ is isomorphic to $S$ whenever there is a ring $S$ and a positive integer $n$ such that $R\left[\left[x_{1}, \ldots, x_{n}\right]\right]$ and $s\left[\left[x_{1}, \ldots, x_{n}\right]\right]$ are isomorphic where $x_{1}, \ldots, x_{n}$ are independent indeterminates over $\mathrm{R}$ and $\mathrm{S}$.

EXAMPLE. If $R$ is a quasi-local ring then so is $R\left[\left[x_{1}, \ldots, X_{n}\right]\right]$ for any positive integer $n$. Since any quasi-local ring is power invariant [7], $R\left[\left[X_{1}, \ldots, X_{n}\right]\right] 1$ s power invariant if $R$ is a quasi-local ring. Then clearly every quasi-local ring is forever-power-invariant.

THEOREM 6. If $R$ is a ring such that $I_{c}(R)$ is $n i l$, then $R$ is forever-powerinvarjant.

PROOF. Suppose that $R$ is a ring such that $I_{c}(R)$ is nil. Let $W=R\left[\left[X_{1}, \ldots, X_{n}\right]\right]$ $=S\left[\left[Y_{1}, \ldots, Y_{n}\right]\right]$. To prove this theorem, it suffices to show that $R$ and $S$ are 1 somorphic. Let $\mathrm{Y}_{1}=a_{0}^{(i)}+\mathrm{X}_{1} \mathrm{U}_{1}^{(i)}+\ldots+\mathrm{X}_{\mathrm{n}} \mathrm{U}_{\mathrm{n}}^{(i)}$ and $x_{i}=b_{0}^{(i)}+Y_{1} v_{1}^{(i)}+\ldots+Y_{n} v_{n}^{(i)}$ for each $i=1, \ldots, n$ where $U_{k}^{(i)}$ and $v_{k}^{(1)}$ are elements of $W$ for each $i=1, \ldots, n$ and $k=1, \ldots, n$ and $a_{0}{ }^{(1)} \epsilon, b_{0}(1) \epsilon S$ for each $i=1, \ldots, n$. Since $\left(W,\left(Y_{i}\right)\right)$ is a complete Hausdorff space, there is a R-homomorphism $\phi$ of $R\left[\left[X_{1}\right]\right]$ into $R\left[\left[X_{1}, \ldots, X_{n}\right]\right]$ such that $\phi\left(X_{1}\right)=\left(Y_{i}\right)=a_{0}^{(i)}+X_{1} U_{1}^{(i)}+\ldots+$ $X_{n} U_{n}{ }^{(i)}$. Then by Theorem 4, $a_{0}{ }^{(i)} \in I_{c}(R)$ for each $1=1, \ldots, n$ and so $a_{0}$ (i) are nilpotent for each $i=1, \ldots, n$. The relation defined between $Y_{1} s$ and $X_{1} s$ yields the following:

$$
\begin{aligned}
Y_{i}= & a_{0}^{(i)}+{ }_{k=1}^{n} b_{0}^{(k)} U_{k}(i)+\left(\sum_{k=1}^{n} V_{1}^{(k)} U_{k}^{(i)}\right) Y_{1}+\ldots \\
& +\left({ }_{k=1}^{n} V_{i}{ }^{(k)} U_{k}^{(i)}\right) Y_{i}+\ldots+\left({ }_{k=1}^{n} v_{n}{ }^{(k)} U_{k}{ }^{(1)}\right) Y_{n}
\end{aligned}
$$

Let $a_{0}^{(i)}={ }_{k=0}^{\infty} C_{k}^{(i)}$ be a homogenous decomposition in $S\left[\left[Y_{1}, \ldots, Y_{n}\right]\right]$. Then since 
$a_{0}{ }^{(i)}$ is nilpotent, $c_{k}{ }^{(i)}$ is nilpotent for each $k=1, \ldots, n$. Let $c_{1}{ }^{(i)}=c_{11}{ }^{(1)} Y_{1}+$ $\ldots+c_{1 n}{ }^{(i)} Y_{n}$, then $c_{1 j}{ }^{(i)}$ is a nilpotent element of $s$ for each $j=1, \ldots, n$. Let $U_{k}^{(i)}=\stackrel{\sum}{=}=0_{U_{k j}}^{(i)}$ and $v_{k}^{(i)}=\sum_{j=0}^{\infty} V_{k j}{ }^{(i)}$ be homogeneous decompositions of elements $\mathrm{U}_{\mathrm{k}}{ }^{(i)}$ and $\mathrm{v}_{\mathrm{k}}{ }^{(i)}$ in $\mathrm{s}\left[\left[\mathrm{Y}_{1}, \ldots, \mathrm{Y}_{\mathrm{n}}\right]\right]$ and let $\mathrm{U}_{\mathrm{kl}}{ }^{(i)}=u_{k 11} \mathrm{Y}_{1}+\ldots+\mathrm{u}_{\mathrm{k} 1 \mathrm{n}} \mathrm{Y}_{\mathrm{n}}$ and $\mathrm{v}_{\mathrm{k} 1}{ }^{(i)}=$ $v_{k 11} Y_{1}+\ldots+v_{k 1 n} Y_{n}$. Then the $Y_{j}$ coefficient of the right side of (1) is

$$
c_{1 j}^{(i)}+\sum_{k=1}^{n} b_{0}^{(k)} u_{k 1 j}{ }^{(i)}+\sum_{k=1}^{p} v_{j 0}^{(k)} u_{k 0}^{(i)}
$$

which is equal to 1 if $j=i$, otherwise, 0 . Since $c_{1 j}$ (i) is nilpotent and

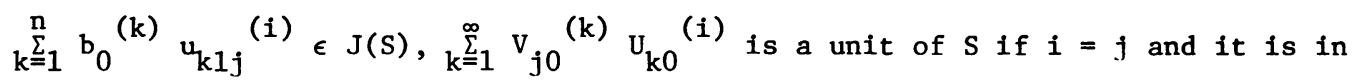
$J(S)$ if $i \neq j$. Let $A=\left(v_{j 0}{ }^{(k)}\right)_{j k}$ and $B=\left(U_{k 0}{ }^{(i)}\right)_{k i}$ be $n \times n$ matrices over $s$, then $A B=\left({ }_{k=1}^{n} v_{j 0}(k) U_{k 0}(i)\right)_{j i}$ in which every diagonal entry is a unit of $S$ and the rest of entries are elements of $J(S)$. So $A B$ is invertible in $M_{n}(S)$ : therefore, both $A$ and $B$ are invertible in $M_{n}(S)$. Clearly, $\left(W,\left(X_{1}, \ldots, x_{n}\right)\right)$ is a complete Hausdorff space. Recall that the linear homogeneous component of $x_{i}=b_{0}{ }^{(i)}$ $+\mathrm{Y}_{1} \mathrm{~V}_{1}{ }^{(i)}+\ldots+\mathrm{Y}_{\mathrm{n}} \mathrm{V}_{\mathrm{n}}{ }^{(\mathrm{i})}$ considered as an element of $\mathrm{s}\left[\left[\mathrm{Y}_{1}, \ldots, \mathrm{Y}_{\mathrm{n}}\right]\right]$ is $\mathrm{Y}_{1} \mathrm{~V}_{10}{ }^{(i)}+\ldots$ $+\mathrm{Y}_{\mathrm{n}} \mathrm{V}_{\mathrm{n} 0}^{(i)}$ for each $i=1, \ldots, n$ and the $n \times n$ matrix $A=\left(\mathrm{V}_{j 0}(i)\right)_{j i}$ is invertible in $M_{n}(S)$. Then by Theorem 2 and Proposition 3, there is an S-automorphism $\psi$ of $S\left[\left[Y_{1}, \ldots, Y_{n}\right]\right]$ such that $\psi\left(Y_{i}\right)=b_{0}{ }^{(i)}+Y_{1} V_{1}{ }^{(i)}+\ldots+Y_{n} V_{n}{ }^{(i)}$ for each $i=1, \ldots, n$. Then $s \cong s\left[\left[Y_{1}, \ldots, Y_{n}\right]\right] /\left(Y_{1}, \ldots, Y_{n}\right) \cong W /\left(\psi\left(Y_{1}\right), \ldots, \psi\left(Y_{n}\right)\right)=W /\left(X_{1}, \ldots, X_{n}\right)$ $=R\left[\left[x_{1}, \ldots, x_{n}\right]\right] /\left(x_{1}, \ldots, x_{n}\right) \cong R$. This completes the proof.

CORROLLARY 7 . If $R\left[t_{1}, \ldots, t_{n}\right]$ is the polynomial ring in indeterminates $t_{1}, \ldots, t_{n}$ over a ring $R$, then it is a forever-power-invariant.

It is easy to see that if $R$ is a ring such that $R\left[\left[X_{1}, \ldots, X_{n}\right]\right]$ is power invariant for any positive integer $n$. Then $R$ is forever-power-invariant. This raises the following open question: If $R$ is a ring such that $I_{c}(R)$ is nil then for any positive integer, is $R\left[\left[\mathrm{X}_{1}, \ldots, \mathrm{X}_{\mathrm{n}}\right]\right]$ power invariant? 


\section{REFERENCES}

1. S. Abhyanker, W. Heinzer, and P. Eakin, On the uniqueness of the coefficient ring in polynomial rings, J. Algebra 23 (1972), 310-342.

2. N. Bourbaki, Algebrè Commutative, Chap. 3 and 7, Paris, Hermann, 1965.

3. D. Coleman and E. Enochs, Isomorphic polynomial rings, Proc. Amer. Math. Soc. 27 (1971), 247-252.

4. P. Eakin and A. Sathaye, R-endomorphisms of $\mathrm{R}[\mathrm{X}]]$ are essentially continuous, Pacific J. Math. 66 (1976), 83-87.

5. R. Gilmer and M. O'Malley, R-endomorphisms of $\mathrm{R}\left[\left[\mathrm{X}_{1}, \ldots, \mathrm{X}_{\mathrm{n}}\right]\right]$, J.Algebra $\underline{48}$ (1977), 30-45.

6. R. Gilmer and M. O'Ma1ley, On R-homomorphisms of power series rings, Advances in Math., (to appear).

7. E. Hamann, On power-invariance, Pacific J. Math., 61 (1975), 153-159.

8. M. Hochster, Non-uniqueness of coefficient rings in a polynomial ring, Proc. Amer. Math. Soc., 34 (1972), 81-82.

9. I. Kaplansky, Commutative rings, Boston, Allyn and Bacon, 1970.

10. J. H. Kim, Power invariant rings, Pacific J. Math. 51 (1974), 207-213.

11. J. H. Kim, R-automorphisms of $\mathrm{R}[\mathrm{t}]\left[\left[\mathrm{X}_{1}, \ldots, \mathrm{X}_{\mathrm{n}}\right]\right]$, J. Algebra (to appear).

12. M. Nagata, Local Rings, New York, Interscience, 1962.

13. M. O'Ma11ey, Isomorphic power series rings, Pacific J. Math., 41 (1972), 503-512.

14. M. O'Malley, R-automorphisms of $\mathrm{R}[\mathrm{X}]]$, Proc. London Math. Soc., 20 (1970), 60-78.

15. M. O'Ma1ley and C. Wood, R-endomorphisms of R[[X]], J. Algebra, 15 (1970), 314-327.

16. 0. Zariski and P. Samue1, Commutative Algebra, Vo1.II, Princeton, N.J.: Van Nostrand, 1960. 


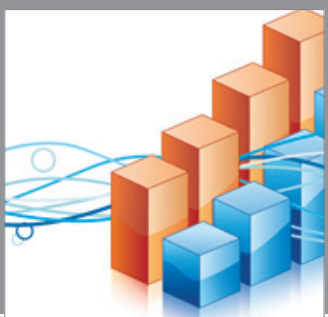

Advances in

Operations Research

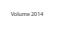

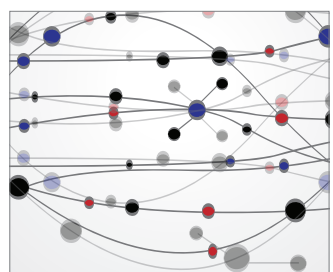

\section{The Scientific} World Journal
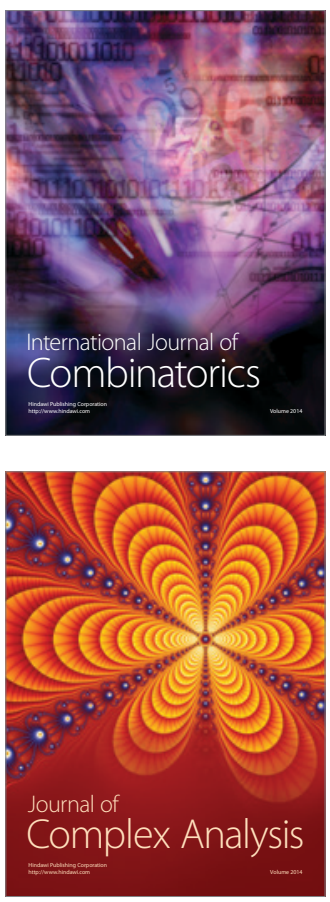

International Journal of

Mathematics and

Mathematical

Sciences
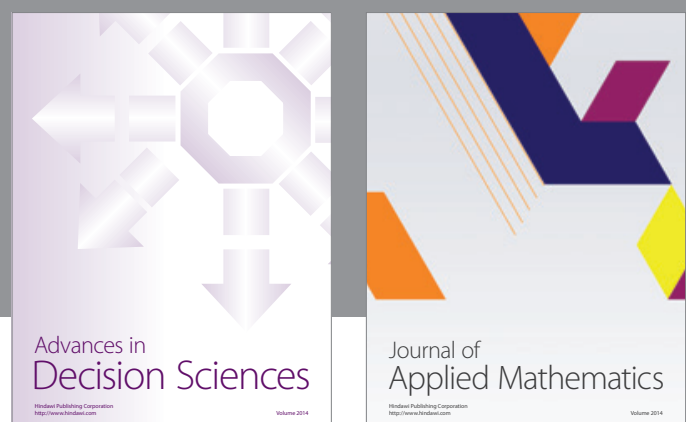

Journal of

Applied Mathematics
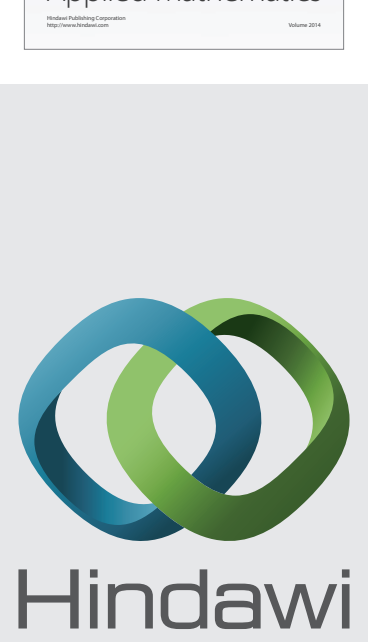

Submit your manuscripts at http://www.hindawi.com
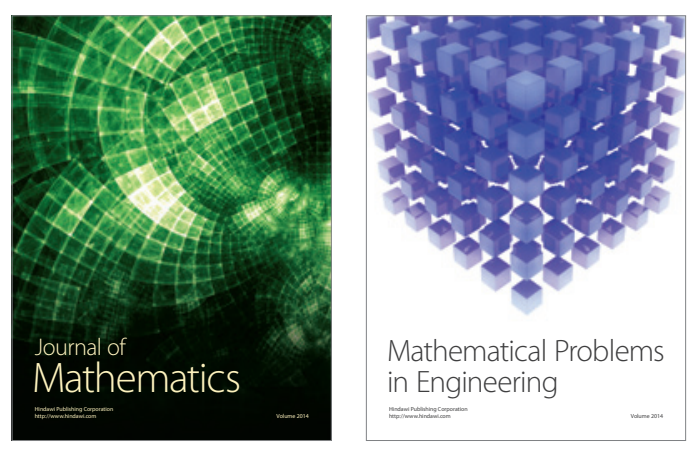

Mathematical Problems in Engineering
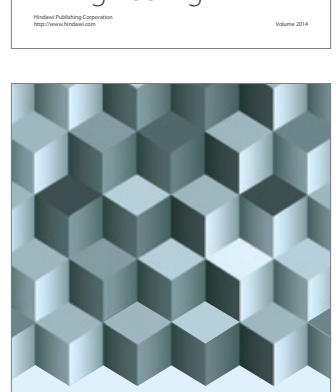

Journal of

Function Spaces
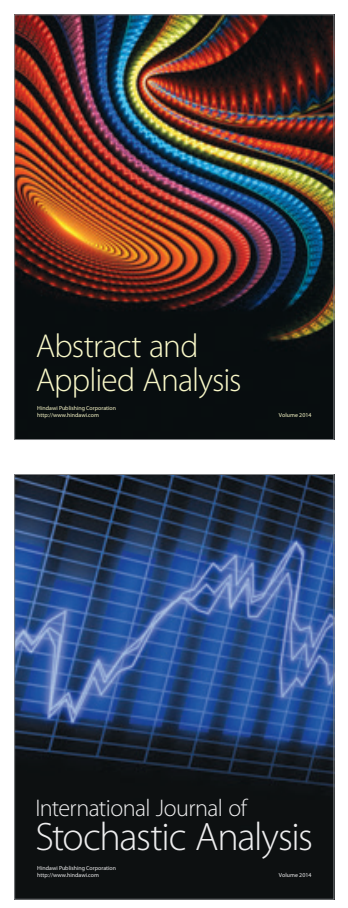

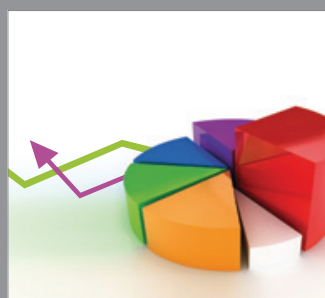

ournal of

Probability and Statistics

Promensencen
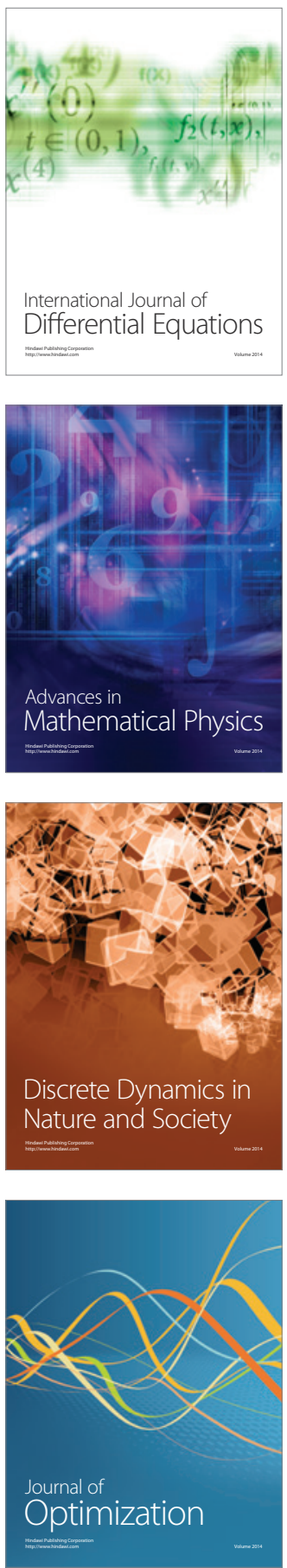